\title{
Changing prevalence of current asthma and inhaled corticosteroid treatment in the UK: population-based cohort 2006-2016
}

\author{
Chloe I. Bloom ${ }^{1}$, Sejal Saglani ${ }^{2}$, Johanna Feary $^{1}$, Debbie Jarvis ${ }^{1}$ and \\ Jennifer K. Quint ${ }^{1}$
} Affiliations: ${ }^{1}$ Population Health and Occupational Disease, National Heart and Lung Institute, Imperial College
London, London, UK. ${ }^{2}$ Paediatric Severe Asthma Group, Inflammation, Repair and Development, National
Heart and Lung Institute, Imperial College London, London, UK.

Correspondence: Chloe I. Bloom, National Heart and Lung Institute, Imperial College London, 1b Manresa Road, London, SW3 6LR, UK. E-mail: chloe.bloom06aimperial.ac.uk

@ERSpublications

Asthma management is changing, with increasing prescriptions of maintenance inhalers and oral corticosteroids. Disease prevalence is decreasing in those aged $<45$ years, with the most rapid decline found in preschool children. http://ow.ly/8g8D30nlBUW

Cite this article as: Bloom CI, Saglani S, Feary J, et al. Changing prevalence of current asthma and inhaled corticosteroid treatment in the UK: population-based cohort 2006-2016. Eur Respir J 2019; 53: 1802130 [https://doi.org/10.1183/13993003.02130-2018].

ABSTRACT Asthma is the most common respiratory disorder in the UK, yet we have incomplete knowledge on the prevalence of current disease, treatment and exacerbations.

We used UK electronic healthcare records, 2006-2016, to estimate the prevalence of current asthma by year, sex and age $(<5,5-11,12-17,18-24,25-54$ and $\geqslant 55$ years), and the proportion prescribed inhaled corticosteroids (ICS) and additional asthma therapy, treated for exacerbations and other asthma care markers.

Overall current asthma prevalence was $6.5 \%$ in 2016 (7.2\% in 2006). Prevalence fell in those aged $<45$ years. The lowest prevalence and largest absolute decrease was in children aged $<5$ years. In $2016,80 \%$ of current asthma patients were managed on ICS (65\% in 2006); this increase occurred in all age groups, primarily due to an increase in low-dose ICS. During this time there was an increase in all age groups in the proportion prescribed additional asthma therapy, treated for an exacerbation within primary care and given an annual asthma review or management plan. Hospitalised exacerbations showed minimal change over time.

Asthma remains highly prevalent and a significant healthcare burden. In those with a diagnosis, there was an increase in ICS prescriptions and treatment of exacerbations across all age groups. This may reflect a trend towards more aggressive asthma management within primary care. An apparent decline in prevalence was observed in those aged $<45$ years, particularly in children aged $<5$ years. 


\section{Introduction}

Surveys of asthma prevalence have shown that the UK has one of the highest national rates of self-reported adult and childhood asthma in Europe [1,2]. Owing to the considerable cost of treatment (inhalers are some of the most expensive drugs in the National Health Service (NHS) budget) and high prevalence, asthma causes a significant healthcare burden in the UK $[3,4]$. To understand the current healthcare burden of asthma, and the projected future burden, it is important to capture recent temporal trends in prevalence of current disease, exacerbations and disease management. Several methods have been used to estimate asthma prevalence, each with their own strengths and limitations. One commonly used method is surveys, but these usually only cover geographically discrete areas, are relatively small in size, focus on specific age groups and rely on self-reporting of symptoms or doctor diagnoses. Many global surveys include a few specific locations in the UK (e.g. the International Study of Asthma and Allergies in Childhood) but the only surveys explicitly carried out across the UK are the National Health surveys (including Health Survey for England, and the Scottish, Welsh and Northern Ireland Health Surveys).

An alternative source from which to estimate prevalence is routinely collected electronic healthcare records (EHRs), which provide data on doctor-diagnosed asthma. This resource is potentially useful for generating estimates of current asthma prevalence because information on doctor diagnosis of symptoms is recorded at the time of the general practitioner (GP) consultation. It is less suitable for estimates of lifetime ("ever") prevalence owing to a lack of complete lifetime data. Because many children with asthma go into remission, and some adults develop asthma de novo, this distinction is relevant with respect to current healthcare burden [5-10]. Another advantage of EHRs is that they can provide accurate data on GP prescriptions.

The aim of this study was to use longitudinal EHRs to estimate the prevalence of doctor-diagnosed current asthma, asthma medication prescriptions and asthma exacerbations between 2006 and 2016. To the authors' best knowledge, this study involves the single largest database used to estimate prevalence, and treatment, of asthma in the UK.

\section{Methods}

\section{Data sources}

Clinical Practice Research Datalink (CPRD) is one of the world's largest databases of longitudinal healthcare records, collecting patient data from a network of GP practices across the UK. It covers 9\% of the population and is nationally representative for age, sex, ethnicity and body mass index [11-13]; just over $60 \%$ of practices have patient-level linkage to Hospital Episode Statistics (HES) data. HES contains data (collected during a visit to an English NHS hospital) on admission date and reason for admission, using International Classification of Diseases 10th revision (ICD-10) codes. In this study, data on all hospital admissions for asthma were collected.

\section{Study population to determine prevalence denominator}

The study population was the whole of CPRD that had research-acceptable healthcare records, according to CPRD quality control, contributing data on a specific date in each year between January 1, 2006 and December 31, 2016. Of the 727 practices contributing data, 325 (45\%) continuously contributed throughout the study period; these practices have been termed "loyal practices".

\section{Asthma population}

Asthma patients were identified using validated asthma Read codes (the clinical terminology system used by GP practices and CPRD) [14]; infants with preschool wheeze were therefore not included as having asthma unless their condition was specifically labelled as asthma. Asthma patients with a co-diagnosis of chronic obstructive pulmonary disease (COPD) were identified using validated Read codes and excluded [15]. Patient data were only eligible at the latest date of 1) becoming research acceptable according to CPRD quality control; 2) being continuously in a CPRD practice; and 3) having their first asthma Read code. Patients stopped contributing data at the earliest date of 1) date of death; 2) date of transfer out of CPRD practice; and 3) practice's last collection date or their "last current asthma date". An age-dependent assumption was made to define when a patient no longer had current asthma, based on their last contact with the GP practice with respect to their asthma, and their last asthma prescription date (if receiving medication). Therefore, the "last current asthma date" was defined as the latest of either 1) the patient's last specific asthma Read code date ( +12 months if aged $5-18$ years; +24 months if aged $\geqslant 18$ years); or 2 ) the patient's last asthma medication prescription date $(+6$ months if aged $<5$ years; +12 months if aged $\geqslant 5$ years). Asthma medications included were short-acting $\beta$-agonist (SABA), inhaled corticosteroid (ICS), long-acting $\beta 2$-agonist (LABA), combination LABA-ICS, leukotriene receptor antagonist (LTRA) or theophylline. 
Other variables

The main asthma guideline used in the UK is the British Thoracic Society (BTS) and Scottish Intercollegiate Guidelines Network (SIGN) British Guideline on the Management of Asthma [16]. Age groups were divided according to the categories used in these guidelines: $<5$ years, $5-11$ years, adolescents and adults. Adults were further divided into two categories owing to the possible impact of COPD after the age of 55 years. For more detailed prevalence estimates, the adult cohort was further broken down by 10 -year age groups.

ICS included single component ICS or LABA-ICS combinations; the dose was defined as the maximum inhaler dose prescribed. The 2016 BTS asthma guidelines were used to define the categorisation of ICS dosage; categories were fine dose beclometasone dipropionate equivalent: very low dose $(<200 \mu \mathrm{g})$, low dose $(200-400 \mu \mathrm{g}$ for children, $\leqslant 799 \mu \mathrm{g}$ for adolescents/adults), medium dose (800-1599 $\mu \mathrm{g})$ and high dose $(\geqslant 1600 \mu \mathrm{g}$ ) (table 9, adults and adolescents inhaler doses, and table 10, children under 10 years old inhaler doses in [16]). The cut-off for an infrequent ICS prescription was arbitrarily defined as two or fewer prescriptions per year. Add-on therapy was defined as any of the following: LABA, LTRA, long-acting muscarinic antagonist (LAMA) or theophylline. An annual asthma review and an asthma management plan were identified using relevant Read codes (available on request).

Asthma exacerbations were categorised as 1) treated within primary care with a short course of oral corticosteroids (not during an annual review or rescue packs); or 2) requiring an overnight hospital stay (identified using HES data). Asthma patients only seen in accident and emergency departments were not included because these data were not complete during the study period.

\section{Prevalence calculation}

Point prevalence was calculated by dividing the numerator by the denominator. The annual numerator was the total number of patients at a randomly set date (February 1) who had 1) their first validated asthma Read code on or before that date; 2) their "last current asthma date" on or after that date; and 3) did not have COPD on or before that date. The denominator was all research-acceptable patients in CPRD on that set date. A sensitivity analysis was performed including patients with a COPD diagnosis. A sensitivity analysis was performed including only loyal GP practices. Confidence intervals were calculated and are shown in the supplementary tables. Linear regression was used to test the statistical significance of change in annual prevalence. All analyses were conducted using Stata version 14 (College Station, TX, USA).

\section{Ethical approval}

The protocol for this research was approved by the Independent Scientific Advisory Committee (ISAC) for Medicines and Healthcare products Regulatory Agency Database Research (protocol 18_055R); the approved protocol was made available during peer review. Generic ethical approval for observational research using the CPRD with approval from ISAC was granted by a Health Research Authority Research Ethics Committee (East Midlands - Derby, reference number 05/MRE04/87). Linked pseudonymised data were provided for this study by CPRD. Data are linked by NHS Digital, the statutory trusted third party for linking data, using identifiable data held only by NHS Digital. Select general practices consented to this process at a practice level with individual patients having the right to opt-out.

\section{Results}

\section{Prevalence of current asthma}

The prevalence of current asthma was $7.2 \%$ in 2006, gradually falling to $6.5 \%$ in 2016 (figure 1 and supplementary table S1). The overall prevalence was lower in males than in females (2016: males 5.8\%,

FIGURE 1 UK current asthma prevalence.

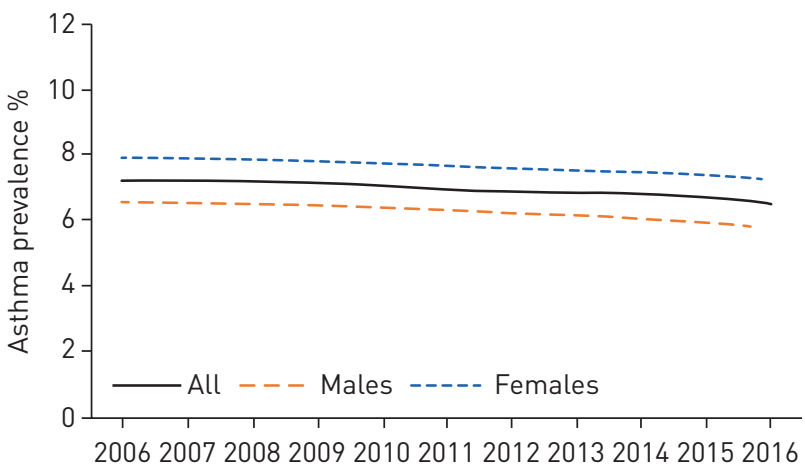


females $7.2 \%$ ) and decreased in both sexes over the study period. Differences in prevalence by sex were dependent on age; prevalence was higher in males until adolescence, after which it was higher in females (figure 2).

In 2016, the group aged $<5$ years had the lowest prevalence of current asthma (1.6\%) and the group aged 12-17 years had the highest prevalence of current asthma (8.0\%) (figure 3 and supplementary table S2). Prevalence gradually fell over the 10 -year period in all age groups, except in those aged $\geqslant 55$ years where it slightly increased. The trend over time was similar in male and female asthma patients in all age groups (supplementary figure S1). Further breakdown into 10-year age groups showed that the prevalence declined in each age group over time, up to the group aged 45-54 years; in all age groups thereafter prevalence slightly increased (supplementary figure S2). The steepest decline occurred in the group aged $<5$ years; the decline in the group aged 25-54 years was minimal (figure 3).

Prevalence estimates in only loyal GP practices exhibited the same magnitude and trends as estimates in all GP practices (supplementary tables S1 and S2). Including patients with a COPD diagnosis resulted in a higher prevalence, as would be expected. It also resulted in a trend for a temporal decline, the opposite of when COPD patients were excluded ( $\geqslant 55$ years: $2006,8.6 \% ; 2016,8.3 \%$; supplementary table S3).

\section{Changes in asthma management}

The percentage of current asthma patients prescribed an ICS in 2006 was 65\%, compared with $80 \%$ in 2016. Throughout follow-up, the groups aged $<5$ years and $\geqslant 55$ years had the highest proportion managed on an ICS, with the lowest proportion in the group aged 12-17 years. The proportion prescribed an ICS increased over time, most markedly in the $<5$ years group and least in the $\geqslant 55$ years group (relative increase between 2006 and 2016: $<5$ years, 47.5\%; 5-11 years, 30\%; 12-17 years, $27.7 \%$; $18-54$ years, 26.5\%; $\geqslant 55$ years, $20.7 \%$ ) (figure 4 and supplementary table S4). There was very little difference in percentages between patients from loyal GP practices and from all GP practices (supplementary table S4). Sex had little effect on receiving ICS or not, or the dose prescribed (data not shown).

The proportion of patients receiving infrequent ICS prescriptions (two or more per year) fell over time and was highest in the groups aged 12-17 years and 5-11 years (supplementary figure S3).

The proportion being prescribed asthma add-on therapy increased in all age groups; this increase occurred for all add-on therapies, except theophylline, which remained stable (supplementary figure S4).

There was a gradual increase in all age groups in the percentage having an annual asthma review (2006, $38.6 \% ; 2016,43.7 \%)$ or receiving an asthma management plan (2006, 6.2\%; 2016, 19.7\%) (supplementary figure S5).

\section{Temporal changes in exacerbations}

The proportion of exacerbations treated in primary care increased over time. The biggest rise occurred in the group aged $<5$ years and the smallest increase in the group aged $\geqslant 55$ years; results were comparable between all GP practices and only loyal GP practices (figure 5 and supplementary table S5). There was little change in the proportion of overnight hospitalised exacerbations, except for an increase in children aged $<5$ years (supplementary figure S6).

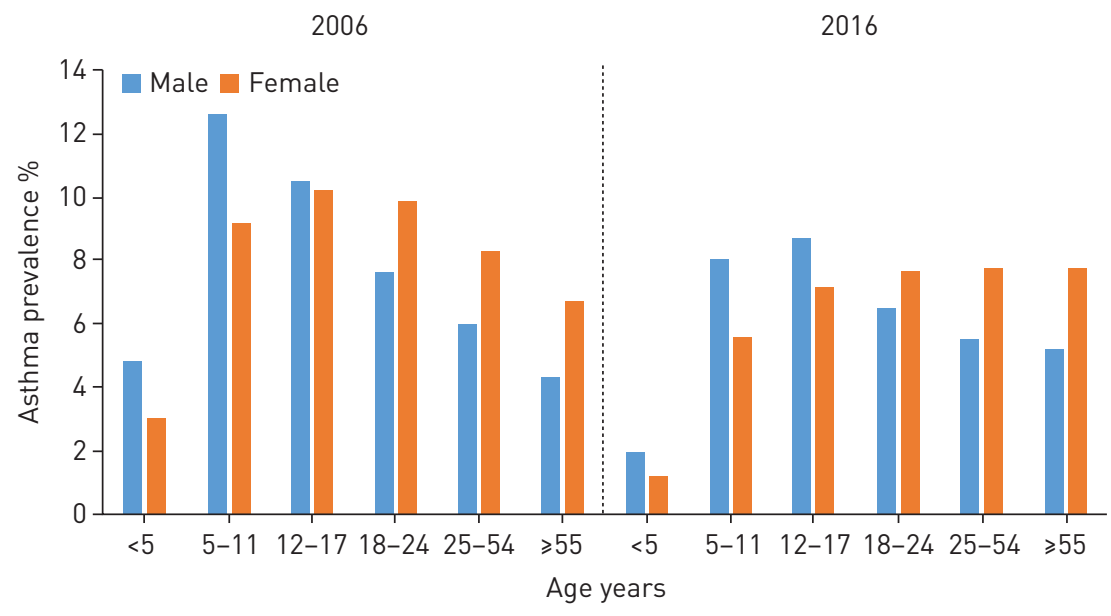

FIGURE 2 Asthma prevalence by age and sex in 2006 and 2016. 
FIGURE 3 Asthma prevalence by age groups.

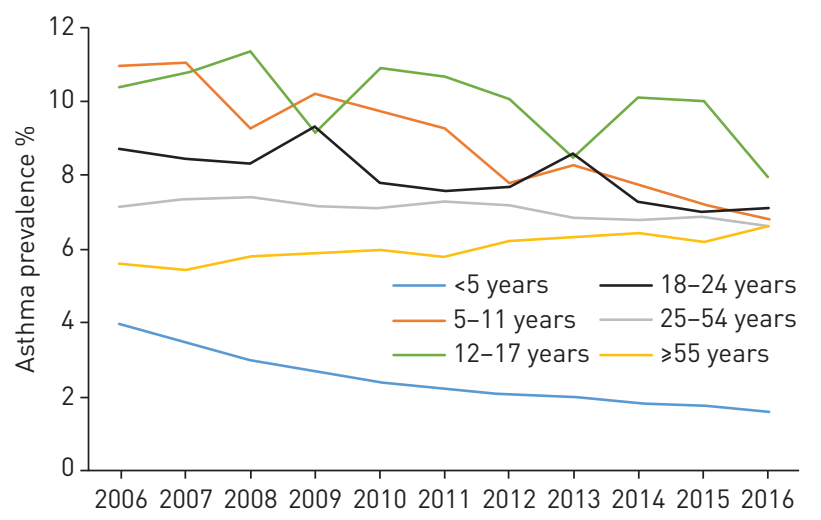

\section{Discussion}

Our study shows that in 2016, just under $6.5 \%$ of the CPRD population excluding COPD patients had current asthma (7.1\% including COPD patients). The prevalence had declined slightly over the previous 10 years, most notably in patients aged $<5$ years, with little change over time in patients aged between 25 and 54 years. In keeping with previous findings, we also found that asthma was more prevalent in males up until around puberty, after which it was more prevalent in females $[17,18,19,20]$.

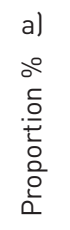
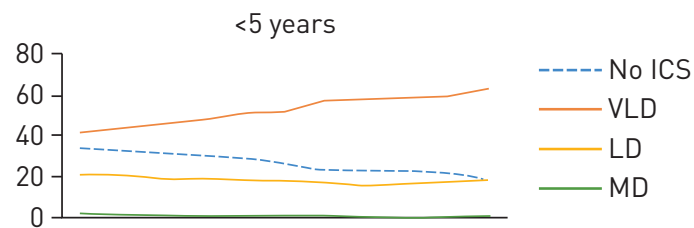

b)

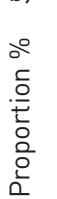
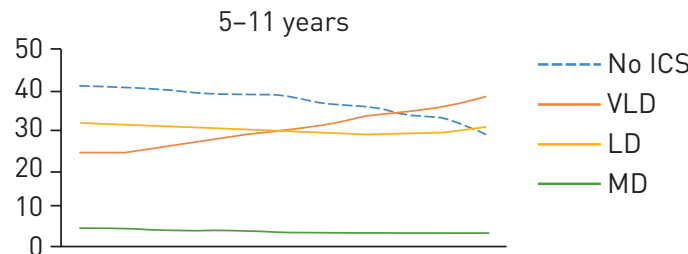

- VLD

- LD

MD

c)

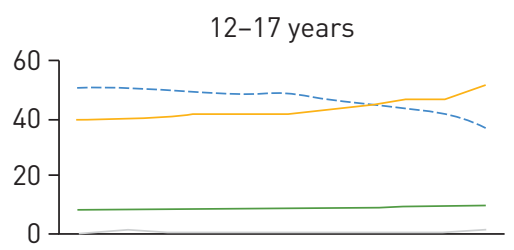

----- No ICS

LD

$-M D$

$H D$

d)

18-54 years

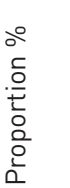

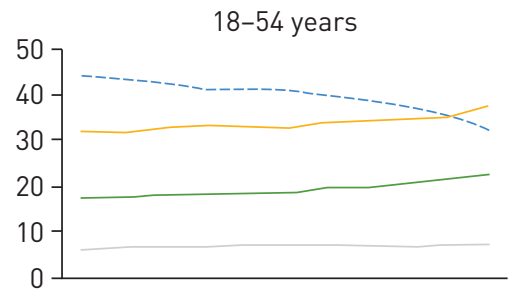

----- No ICS

LD

MD

$H D$

e)

FIGURE 4 Temporal changes of proportion in each inhaled corticosteroid (ICS) dose category by age. VLD: very low dose $(<200 \mu \mathrm{g})$; LD: low dose $1200-400 \mu \mathrm{g}$ for children, $<800 \mu \mathrm{g}$ for adolescents/adults); MD: medium dose (800-1599 $\mu \mathrm{g})$; HD: high dose $(\geqslant 1600 \mu \mathrm{g})$.

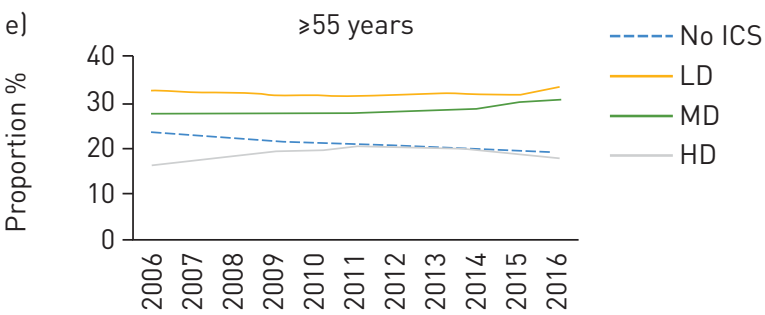


FIGURE 5 Temporal changes of exacerbations over time and by age. Proportion of patients with at least one exacerbation treated within primary care in the year shown.

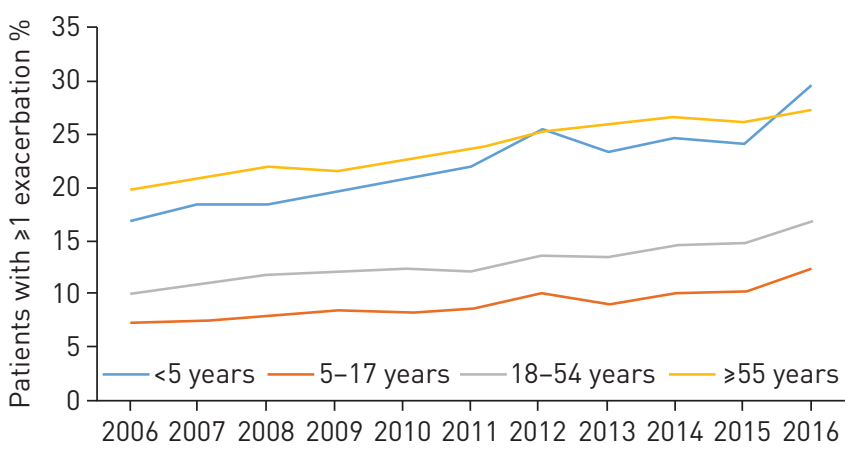

\section{Comparing findings to previous studies}

Two previous studies used primary care EHR data to estimate the lifetime ("ever") prevalence of doctor-diagnosed asthma in England (2001-2005) and the UK (2004-2012) [18, 21]. Both these studies reported a steady increase in lifetime prevalence in those aged $>16$ years, but a decrease in incident asthma in all ages. These studies used information from databases that were unlikely to cover the entire lifetime of the individuals included, so are likely to have underestimated the true lifetime prevalence. This might explain why their lifetime estimates were only $3-4 \%$ higher than our current prevalence estimates.

Survey data (using self-report of symptoms, doctor diagnosis and treatment) from the UK have found approximately double the prevalence of "asthma" in all age groups compared to our estimates [3, 19, 21-27]. However, the four established asthma surveys that have been used in the UK define asthma by the presence of self-reported wheeze, which may be related to other factors (interpretation of "wheeze") and other pathologies, thus overestimating the presence of clinical asthma. Furthermore, changes in prevalence of wheeze might be correlated with increased symptom awareness, better education or augmented readiness to report wheeze [28]. Surveys might also overestimate the prevalence of disease because of low response rates, which might bias the study (patients with symptoms may be more likely to respond to a survey than those without) [29].

Unlike surveys, which are often only collected from specific centres and are therefore not generalisable to the whole of the UK $[19,22,23,25,27]$, this study has wide national geographical coverage with prevalence estimates based on large samples. Lastly, previous surveys and EHR data that included older patients did not address the difficulty of misclassification with COPD. Interestingly, we found an increasing prevalence of asthma-only diagnoses in older patients, whereas asthma with or without a COPD diagnosis showed decreasing prevalence. One explanation might be that primary care physicians are less likely now to label all obstructive airways disease in older patients as COPD, perhaps owing to increasing awareness of late-onset adult asthma or increased use of the lower limit of predicted normal in spirometry [30].

\section{Comparison to recent European estimates}

Unlike this study, other studies with up-to-date estimates of prevalence in Western European countries have found a rise in current asthma prevalence over the past 10 years. The latest estimates for Sweden were derived from repeated cross-sectional self-reporting surveys, and found an increase from $9.9 \%$ to $10.6 \%$ in adult asthma between 2006 and 2016, although it is notable that only around half of those surveyed responded [31]. The most recent prevalence estimates for Finland, of persistent asthma only, obtained from a social insurance registry showed an increase between 2000 and 2013 of $3.6 \%$ to $4.4 \%$; this could be related to the increased recognition of disease owing to their national asthma programme [32].

\section{Strengths and limitations of EHR data}

Studies using survey data of asthma symptoms as compared to EHR data of doctor-diagnosed asthma provide information on different aspects, each with their own advantages and disadvantages. Two key advantages of EHR data are the probable increase in diagnostic accuracy and a reduction in recall bias, although there still remains potential for misclassification due to the clinical difficulty in diagnosing asthma in primary care, particularly in young children and adult smokers. Another limitation of EHR data is that some people may not report their symptoms to their doctor, due to an unwillingness or a lack of perception of the meaning of their symptoms; this is a distinct advantage of self-reporting of wheeze in survey data. Owing to the complexity of defining asthma, and the lack of a gold-standard diagnostic test, patients may be under- or over-diagnosed. Factors associated with under-diagnosis in primary care include underreporting of symptoms, poor diagnostic sensitivity of spirometry and lack of access to high-specificity diagnostic tests [30]; this diagnostic challenge is potentially most apparent in the youngest and oldest populations. 
Limitations of this study

We have used a validated definition of asthma, but had to make assumptions to define current asthma (based on the patient's last Read code and asthma medication prescription). It is probable our case definition did not identify all individuals with current asthma; missed individuals were likely to have such mild current asthma that little doctor contact had occurred. In the UK, the Quality and Outcomes Framework (QOF) is a system used to financially reward GPs for implementing good practice; changes in these QOFs over time could influence GP coding. Fortunately, the QOFs for asthma did not change over the study period. Reassuringly, prevalence and treatment trends were the same for practices continuously in CPRD and those that entered CPRD later during the study period; however, it is possible CPRD practices in general are more up-to-date in terms of clinical practice and following of guideline recommendations than non-CPRD practices.

Another potential limitation was the assumption that all courses of oral corticosteroids prescribed during an annual asthma review (accounting for $<2 \%$ of short courses of corticosteroids) were kept for rescue packs, and therefore excluded in our analysis. It is also not known if any of the prescriptions were actually dispensed or adhered to; the increase in ICS frequency could suggest an increase in adherence or a change in prescription patterns.

\section{Potential explanations for the observed findings}

We observed that the proportion of patients managed on an ICS has increased, alongside an increase in more regular ICS prescriptions, add-on asthma therapy and asthma management plans. This observation suggests more aggressive management of disease within primary care, which is supported by the increase in treatment for exacerbations in primary care. Alternatively, alongside the decrease in prevalence in children and adults aged up to 45 years, these findings could suggest that there has been increasing reticence to give a diagnosis of asthma. Diagnostic pathways in UK asthma guidelines (BTS/SIGN British Guideline on the Management of Asthma) did not change during the study, but rely greatly upon clinical judgement; cultural changes might have led to an increasing reluctance to formally diagnose asthma without higher clinical certainty. The reduction in infrequent ICS prescriptions is conceivably influenced by several factors, including changes over time in adherence or diagnostic improvements. However, it is also in keeping with the other changes observed that suggest alterations in the primary care management of disease and/or labelling of mildly symptomatic patients. The most rapid increase in ICS use was in children aged $<5$ years, which was predominantly in low-dose ICS; this supports the concept that doctors have become disinclined to give an asthma diagnosis to preschoolers with wheeze (perhaps using terms such as "preschool wheeze" instead); thus, those who actually receive a formal diagnosis are more likely to require pharmacological treatment. A less likely explanation for this pattern is that the natural history of asthma has changed in the UK over the past 10 years, such that it now presents in a more severe form.

In 2016, the BTS/SIGN guidelines changed to recommend starting low-dose ICS in newly diagnosed asthma patients; previously, patients were commenced on SABA as needed. Our findings indicate this new approach was starting to infiltrate primary care even before the release of the latest guidelines.

\section{Implications of these findings}

Globally, survey data has been instrumental in our appreciation of asthma epidemiology, but with the availability of large-volume EHR data we can now obtain an alternative, and arguably more relevant, estimation of prevalence when such data are sufficiently and accurately recorded. In this study we have used the largest source of EHR data from the UK to describe changes in asthma prevalence and management over the past 10 years. We have shown that the prevalence of current disease is decreasing in younger age groups. Overall, patients are increasingly managed with ICS and add-on therapies; concurrently, the number of exacerbations treated in primary care has risen. These findings suggest a trend towards more aggressive asthma management, and may indicate a reticence to give a formal asthma diagnosis until a certain level of disease severity.

Conflict of interest: C.I. Bloom has nothing to disclose. S. Saglani has nothing to disclose. J. Feary reports personal fees from Teva, outside the submitted work. D. Jarvis has nothing to disclose. J.K. Quint reports grants and personal fees from AZ, grants from MRC, GSK, BLF, The Health Foundation, Insmed, Bayer and Wellcome, personal fees from Chiesi, personal fees and other (paid to Imperial College) from BI, other (paid to Imperial College) from Quintiles IMS and personal fees (paid to Imperial College) from Teva, outside the submitted work.

\section{References}

1 Janson C, Anto J, Burney P, et al. The European Community Respiratory Health Survey: what are the main results so far? European Community Respiratory Health Survey II. Eur Respir J 2001; 18: 598-611. 
2 Asher MI, Montefort S, Björkstén B, et al. Worldwide time trends in the prevalence of symptoms of asthma, allergic rhinoconjunctivitis, and eczema in childhood: ISAAC Phases One and Three repeat multicountry cross-sectional surveys. Lancet 2006; 368: 733-743.

3 Mukherjee M, Stoddart A, Gupta RP, et al. The epidemiology, healthcare and societal burden and costs of asthma in the UK and its member nations: analyses of standalone and linked national databases. BMC Med 2016; 14: 113.

4 Health \& Social Care Information Centre. Prescription cost analysis England. 2012. www.statisticsauthority.gov.uk/ publication/prescription-cost-analysis-england-2012-health-and-social-care-information-centre/ Date last accessed: July 16, 2018.

5 Beuther DA, Sutherland ER. Overweight, obesity, and incident asthma. Am J Respir Crit Care Med 2007; 175: 661-666.

6 Shaaban R, Zureik M, Soussan D, et al. Rhinitis and onset of asthma: a longitudinal population-based study. Lancet 2008; 372: 1049-1057.

7 Covar RA, Strunk R, Zeiger RS, et al. Predictors of remitting, periodic, and persistent childhood asthma. J Allergy Clin Immunol 2010; 125: 359-366.

8 Fuchs O, Bahmer T, Rabe KF, et al. Asthma transition from childhood into adulthood. Lancet Respir Med 2017; 5: 224-234.

9 Sears MR, Greene JM, Willan AR, et al. A longitudinal, population-based, cohort study of childhood asthma followed to adulthood. N Engl J Med 2003; 349: 1414-1422.

10 Andersson M, Hedman L, Bjerg A, et al. Remission and persistence of asthma followed from 7 to 19 years of age. Pediatrics 2013; 132: e435-e442.

11 Herrett E, Gallagher AM, Bhaskaran K, et al. Data resource profile: Clinical Practice Research Datalink (CPRD) Int J Epidemiol 2015; 44: 827-836.

12 Mathur R, Bhaskaran K, Chaturvedi N, et al. Completeness and usability of ethnicity data in UK-based primary care and hospital databases. J Public Health (Oxf) 2014; 36: 684-692.

13 Bhaskaran K, Forbes HJ, Douglas I, et al. Representativeness and optimal use of body mass index (BMI) in the UK Clinical Practice Research Datalink (CPRD). BMJ Open 2013; 3: e003389.

14 Nissen F, Morales DR, Mullerova $\mathrm{H}$, et al. Validation of asthma recording in the Clinical Practice Research Datalink (CPRD). BMJ Open 2017; 7: e017474.

15 Quint JK, Mullerova H, DiSantostefano RL, et al. Validation of chronic obstructive pulmonary disease recording in the Clinical Practice Research Datalink (CPRD-GOLD). BMJ Open 2014; 4: e005540.

16 British Thoracic Society. BTS/SIGN British guideline on the management of asthma. London, British Thoracic Society, 2016.

17 Bloom CI, Nissen F, Douglas IJ, et al. Exacerbation risk and characterisation of the UK's asthma population from infants to old age. Thorax 2018; 73: 313-320.

18 Respiratory Health of the Nation. Asthma statistics | British Lung Foundation. 2016. https://statistics.blf.org.uk/ asthma Date last accessed: July 16, 2018.

19 Anderson HR, Gupta R, Strachan DP, et al. 50 years of asthma: UK trends from 1955 to 2004. Thorax 2007; 62: 85-90.

20 Schatz M, Camargo CA. The relationship of sex to asthma prevalence, health care utilization, and medications in a large managed care organization. Ann Allergy Asthma Immunol 2003; 91: 553-558.

21 Simpson CR, Sheikh A. Trends in the epidemiology of asthma in England: a national study of 333,294 patients. $J$ R Soc Med 2010; 103: 98-106.

22 Sembajwe G, Cifuentes M, Tak SW, et al. National income, self-reported wheezing and asthma diagnosis from the World Health Survey. Eur Respir J 2010; 35: 279-286.

23 Chinn S, Jarvis D, Burney P, et al. Increase in diagnosed asthma but not in symptoms in the European Community Respiratory Health Survey. Thorax 2004; 59: 646-651.

24 Mukherjee M, Gupta R, Farr A, et al. Estimating the incidence, prevalence and true cost of asthma in the UK: secondary analysis of national stand-alone and linked databases in England, Northern Ireland, Scotland and Wales-a study protocol. BMJ Open 2014; 4: e006647.

25 Gupta R, Sheikh A, Strachan DP, et al. Burden of allergic disease in the UK: secondary analyses of national databases. Clin Exp Allergy 2004; 34: 520-526.

26 To T, Stanojevic S, Moores G, et al. Global asthma prevalence in adults: findings from the cross-sectional world health survey. BMC Public Health 2012; 12: 204.

27 Lai CKW, Beasley R, Crane J, et al. Global variation in the prevalence and severity of asthma symptoms: phase three of the International Study of Asthma and Allergies in Childhood (ISAAC). Thorax 2009; 64: 476-483

28 Barraclough R, Devereux G, Hendrick DJ, et al. Apparent but not real increase in asthma prevalence during the 1990s. Eur Respir J 2002; 20: 826-833.

29 Johannessen A, Verlato G, Benediktsdottir B, et al. Longterm follow-up in European respiratory health studies patterns and implications. BMC Pulm Med 2014; 14: 63.

30 Aaron SD, Boulet LP, Reddel HK, et al. Underdiagnosis and overdiagnosis of asthma. Am J Respir Crit Care Med 2018; 198: 1012-1020.

31 Backman H, Räisänen P, Hedman L, et al. Increased prevalence of allergic asthma from 1996 to 2006 and further to 2016-results from three population surveys. Clin Exp Allergy 2017; 47: 1426-1435.

32 Janson C, Johannessen A, Franklin K, et al. Change in the prevalence asthma, rhinitis and respiratory symptom over a 20 year period: associations to year of birth, life style and sleep related symptoms. BMC Pulm Med 2018; 18: 152 . 